\title{
Tunneling and State Specificity in Unimolecular Reactions
}

\author{
WILLIAM H. MILLER
}

Department of Chemistry, University of California, and the Materials and Molecular Research Division of the Lawrence Berkeley Laboratory, Berkeley, California 94720

Received May 28, 1986 (Revised Manuscript Received June 27, 1986)

\section{Introduction}

In the standard expositions ${ }^{1,2}$ on unimolecular reaction rates there is little discussion of the effect of quantum mechanical tunneling. This is due partly to the fact that traditional ways of studying such reactions (e.g., thermal excitation, cf. the Lindemann mechanism) tend to obscure subtleties of the reaction dynamics such as tunneling, state-specific effects, etc. Also, very seldom has one had much quantitative information about the potential energy surfaces (i.e., barrier heights and other properties of the transition state) for these reactions, so that these parameters have traditionally been adjusted to fit the data, and this can also hide specifics of the dynamics.

Both of these situations are beginning to change, however, as molecular beam and a variety of laser methods make it possible to study unimolecular processes under near-ideal, collisionless conditions, where the molecule of interest is excited to an extremely well-defined initial state and then reacts without other competing processes. ${ }^{3-5}$ These studies require more rigorous dynamical descriptions in order to understand the variety of phenomena seen at this new level of detail. Also, one is beginning to have usefully reliable ab initio quantum chemistry calculations for transitionstate properties of the reacting molecules. ${ }^{6,7}$

The purpose of this paper is to review the role of tunneling in unimolecular reaction rates. (Tunneling in gas-phase species can also manifest itself as splittings of spectral lines-particularly so for symmetric isomerizations - and though not explicitly discussed in this review, this phenomena can also be treated by the theoretical methods described herein. The tunneling splitting in malonaldehyde is an excellent example of this. ${ }^{8,9}$ ) Since the state-specific character of unimolecular reactions is intimately involved with tunneling effects, this feature of unimolecular reactions is also discussed. Though I will try to keep the discussion as generally applicable as possible, it will be useful to illustrate a number of points with the specific unimolecular reaction for which the significance of tunneling

$$
\mathrm{H}_{2} \mathrm{CO} \rightarrow \mathrm{H}_{2}+\mathrm{CO}
$$

and state-specificity have been most strongly established. This is an ideal example because so much detailed experimental information is available, because the potential energy surface (e.g., barrier heights, vibrational frequencies, and other transition-state properties) is known to an exceptional degree and because the dynamics involves hydrogen atom motion which enhances tunneling effects. The reader interested in the complete story on the unimolecular dissociation of

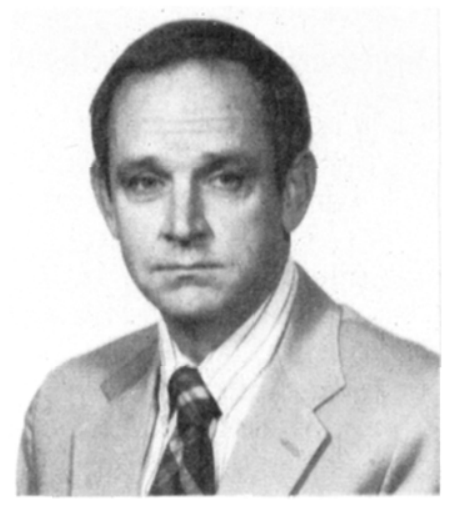

William H. Miller was born in Kosciusko, MI, in 1941. He received a B.S. in Chemistry from Georgia Tech (1963) and a Ph.D. in Chemical Physics from Harvard (1967). Postdoctoral years were spent as a NATO Fellow in Freiburg (Germany) and as a Junior Fellow in the Society of Fellows (Harvard). He joined the Berkeley faculty in 1969 where he has been Professor of Chemistry since 1974. His research has dealt with various aspects of scattering theory and chemical dynamics, most notably with the development of a general semiclassical theory for such processes. He has been a Sloan Fellow, a Dreyfus Scholar, a Guggenheim Fellow, an Overseas Fellow of Churchill College (Cambridge), and a U.S. Senior Scientist Alexander von Humbolt Awardee and in 1974 received the Annual Prize of the International Academy of Quantum Molecular Science. In 1985 he was elected to membership in the above International Academy and also received the Ernest Orlando Lawrence Memorial Award in Chemistry.

formaldehyde should see the recent review by Moore and Weisshaar. ${ }^{4}$

The recent review by $\mathrm{Crim}^{3}$ on unimolecular reactions following selective (usually laser) excitation is also relevant to the topic of this paper, as is the review by Dykstra $^{6}$ on ab initio quantum chemistry calculations of potential barriers for unimolecular rearrangements. Though not dealing specifically with tunneling, the topic of intramolecular vibrational energy redistribution (IVR) in highly vibrationally excited states is relevant to state-specific effects in unimolecular reactions, and there are a number of reviews related to IVR. ${ }^{5,10}$ Also of interest is a series of papers by Kay ${ }^{11}$ on unimolecular reactions, though these are not specifically related to tunneling effects therein.

Tunneling in unimolecular processes is, of course, no different from tunneling in other phenomena (e.g., bimolecular rections), and the same theoretical models are thus used in these various applications. The interested reader should thus take special note of the article in this volume by Schatz ${ }^{12}$ which reviews tunneling effects in bimolecular reactions, which has historically received much greater attention. Also relevant is the recent review by Garrett and Truhlar ${ }^{13}$ which 
discusses tunneling corrections to transition-state theory. One should also note that there are other theoretical descriptions of tunneling that employ diabatic (i.e., crossing) potential energy curves, usually with the golden rule (first-order time-dependent perturbation theory) used to calculate the rate. ${ }^{14}$ This approach is often useful for reactions in condensed phases and when the theory is used in a phenomenological framework; it is generally not useful if taken literally and used in an $\mathrm{ab}$ initio fashion for gas phase reactions.

Section II first discusses tunneling effects within the statistical approximation to unimolecular reactions, and section III discusses state-specific unimolecular rates.

\section{Tunnelling within a Stat/stical Approximation}

The statistical approximation for unimolecular rate constants, i.e., the well-known RRKM theory, ${ }^{1,2,15}$ is microcanonical transition-state theory. To incorporate tunneling effects in RRKM theory, one thus simply incorporates tunneling effects into transition-state theory. ${ }^{16}$ Because it is microcanonical transition-state theory that is relevant, however, rather than the canonical version most familiar for bimolecular rate constants, the tunneling correction is not a simple multiplicative factor as it is in the simplest version of canonical transition state theory.

\section{A. Separable Reaction Coordinate}

The simplest way to incorporate tunneling into transition-state theory is to assume that motion along the reaction coordinate is separable from the other degrees of freedom. The unimolecular rate constant for a molecule with total energy $E$ and total angular momentum $J$ is then given by ${ }^{17,18}$

$$
k(E, J)=[2 \pi \hbar \rho(E, J)]^{-1} \sum_{\mathbf{n}, K} P\left(E-\epsilon_{\mathbf{n} J K}{ }^{*}\right)
$$

where $\left\{\epsilon_{\mathbf{n} J K}{ }^{\ddagger}\right\}$ are the vibrational and rotational energy levels of the "activated complex" in terms of the $(F-$ 1) vibrational quantum numbers $\mathbf{n}=\left(n_{1}, n_{2}, \ldots, n_{F-1}\right)$ and angular momentum quantum numbers $(J, K)$. $(J$ is the conserved total angular momentum quantum number, and $K$ is the nonconserved projection of total angular momentum onto a body-fixed axis.) $P\left(E_{F}\right)$ is the one-dimensional tunneling probability as a function of the energy $E_{F}$ along the reactant coordinate at the transition state. $\rho$ is the density of reactant states per unit energy

$$
\rho(E, J)=\sum_{\mathbf{n}, K} \delta\left(E-\epsilon_{\mathbf{n} J K}\right)
$$

where $\left\{\epsilon_{\mathrm{n} J K}\right\}$ are the energy levels of the reactant molecule in terms of the $F$ vibrational quantum numbers $\mathbf{n}=\left(n_{1}, \ldots, n_{F}\right)$ and angular momentum quantum numbers $(J, K)$.

In practice the energy levels $\epsilon_{\mathbf{n} J K}$ are usually approximated by those of a harmonic oscillator rigid rotor

$$
\epsilon_{\mathbf{n} J K}=\sum_{i=1}^{F} \hbar \omega_{i}\left(n_{i}+1 / 2\right)+A J(J+1)+(C-A) K^{2}
$$

where $\left\{\omega_{i}\right\}$ are the vibrational frequencies of the reactant molecule and $A$ is the average of the two most nearly equal of the three rotation constants and $C$ is the third one.
If the transition state corresponds to a well-defined reaction barrier (i.e., a "tight" transition state), then the energy levels of the activated complex can also be approximated in a similar fashion

$$
\begin{aligned}
\epsilon_{\mathbf{n} J K} & = \\
V_{0} & +\sum_{i=1}^{F-1} \hbar \omega_{i}^{*}\left(n_{i}+1 / 2\right)+A^{*} J(J+1)+\left(C^{\ddagger}-A^{\ddagger}\right) K^{2}
\end{aligned}
$$

where $V_{0}$ is the potential energy and $\left\{\omega_{i}^{\ddagger}\right\}$ and $\left(A^{*}, C^{\ddagger}\right)$ are vibrational frequencies and rotational constants, at the transition-state geometry. If the transition state does not correspond to a well-defined potential energy barrier, e.g., a "loose" transition state, then one must determine $V_{0},\left\{\omega_{i}^{*}\right\}, A^{*}$, and $C^{*}$ as functions of a reaction coordinate and choose the transition state as the location where the rate constant is a minimum..$^{19-21}$ It is also usually necessary in this case to recognize that some of the vibrational modes of the transition state become rotational degrees of freedom of the fragment molecules; it is thus necessary to take anharmonicity of these degrees of freedom into account, by using either hindered rotor energy levels or simply an empirical interpolation formula between the "tight" harmonic energy levels and the "loose" rotational energy levels. ${ }^{22}$

It is easy to see that the classical RRKM expression $^{1,2,15}$ for the rate constant is regained if one ignores tunneling, i.e., replaces the tunneling probability $P\left(E_{F}\right)$ by a step function

$$
P\left(E_{F}\right) \rightarrow h\left(E_{F}\right) \equiv\left\{\begin{array}{l}
1, E_{F}>0 \\
0, E_{F}<0
\end{array}\right.
$$

\section{B. What Is $k(E, J)$}

Before discussing specific examples, it is useful to emphasize the physical meaning of $k(E, J)$, the unimolecular rate constant for a molecule with total energy $E$ and total angular momentum $J$. (For the present discussion $J$ will not be indicated explicitly.) Specifically, $k(E)$ is the average unimolecular rate constant for a molecule with energy $E$ whether the molecule is either (a) intrinsically statistical, i.e., all states with essentially the same energy have essentially the same rate constant, or (b) state specific, i.e., different states with essentially the same total energy have significantly different rate constants. A statistical approximation to the rate will be adequate if either the molecule itself is statistical, case a above, or the experiment is statistical, i.e., averages over many states. To distinguish between cases $a$ and b obviously requires experiments (or theories) that measure (or calculate) rate constants for individual quantum states of the molecule. In either case, though, the microcanonical transition-state theory rate constant of section $\mathrm{A}$ is an approximation to the average rate at energy $E$.

To illustrate the fact that statistical theory yields the correct average rate constant $k(E)$ even if the molecule is intrinsically state-specific, it is useful to consider a completely separable Hamiltonian for a system of two degrees of freedom ${ }^{23}$

$H\left(p_{x}, x, p_{y}, y\right)=\frac{p_{x}{ }^{2}}{2 m}+\frac{p_{y}{ }^{2}}{2 m}+v_{x}(x)+1 / 2 m \omega_{y}{ }^{2} y^{2}$

Mode $y$ is harmonic, but the potential $v_{x}(x)$ is as sketched in Figure $1 ; x$ is thus the reaction coordinate 


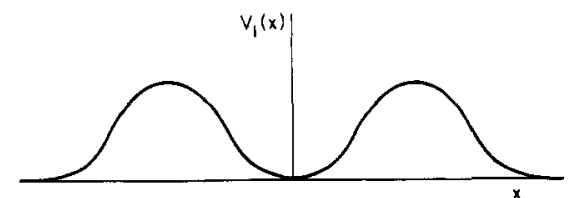

Figure 1. Sketch of the potential $v_{x}(x)$ for the dissociative degree of freedom for the Hamiltonian of eq 2.6.

along which the particle can dissociate by tunneling through the barrier. Because the $x$ and $y$ motions are separable, both $n_{x}$ and $n_{y}$ are good quantum numbers. The energy of the state characterized by quantum numbers $\left(n_{x}, n_{y}\right)$ is

$$
E_{n_{x} n_{y}}=\epsilon_{n_{x}}+\hbar \omega_{y}\left(n_{y}+1 / 2\right)
$$

where $\epsilon_{n_{x}}$ is the energy level of potential $v_{x}$, and the unimolecular decay (i.e., tunneling) rate constant is

$$
k_{n_{x} n_{y}}=\left(\frac{\partial \epsilon_{n_{x}}}{\partial n_{x}} / 2 \pi \hbar\right) P\left(\epsilon_{n_{x}}\right)
$$

Here $P$ is the tunneling probability for the $x$ motion, and $\left(\partial \epsilon_{n_{x}} / \partial n_{x} / 2 \pi \hbar\right)$ is the frequency of motion in the $x$ potential. It is clear that eq 2.7 and 2.8 describe an extremely state-specific situation; i.e., the unimolecular decay rate of eq 2.8 depends only on the energy $\epsilon_{n_{x}}$ in the $x$ mode. Thus states with essentially the same total energy $E_{n_{x} n_{y}}$ can have vastly different rate constants, depending on how much of the total energy is in the reaction coordinate (mode $x$ ). The average rate at total energy $E$ is defined by

$$
k(E) \equiv \frac{\sum_{n_{x}, n_{y}} \delta\left(E-E_{n_{x} n_{y}}\right) k_{n_{x} n_{y}}}{\sum_{n_{x}, n_{y}} \delta\left(E-E_{n_{x} n_{y}}\right)}
$$

where in practice the $\delta$ functions should be broadened somewhat so that at least several states contribute to the sums. Replacing the sum over $n_{x}$ by an integral and using eq 2.7 and 2.8 and the definition of the density $\rho$ of reactant states

$$
\rho(E)=\sum_{n_{x}, n_{y}} \delta\left(E-E_{n_{x} n_{y}}\right)
$$

gives

$$
\begin{aligned}
& k(E)= \\
& \frac{1}{2 \pi \hbar \rho(E)} \sum_{n_{y}} \int \mathrm{d} n_{x} \frac{\partial \epsilon_{n_{x}}}{\partial n_{x}} P\left(\epsilon_{n_{x}}\right) \delta\left(E-\epsilon_{n_{x}}-\hbar \omega_{y}\left(n_{y}+1 / 2\right)\right) \\
& =\frac{1}{2 \pi \hbar \rho(E)} \sum_{n_{y}} P\left(E-\hbar \omega_{y}\left(n_{y}+1 / 2\right)\right)
\end{aligned}
$$

which is recognized as the standard microanonical transition state rate (i.e., eq 2.1) for this example. Thus even for this extreme limit of state specificity, the average rate constant for energy $E$ is seen to be given by statistical theory.

\section{Example: $\mathrm{H}_{2} \mathrm{CO} \rightarrow \mathrm{H}_{2}+\mathrm{CO}$}

Figure 2 shows a sketch of the potential energy surface for the unimolecular decomposition of formaldehyde in its ground electronic state $\left(\mathrm{S}_{0}\right), \mathrm{H}_{2} \mathrm{CO} \rightarrow$ $\mathrm{H}_{2}+\mathrm{CO}$, and Figure 3 shows the unimolecular rate
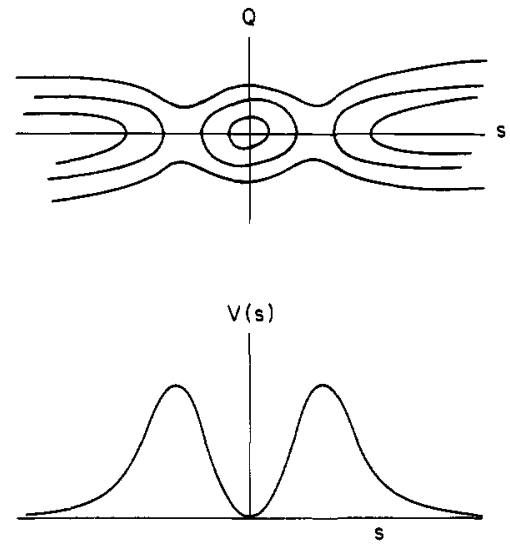

Figure 2. Sketch of the potential energy surface for the formaldehyde dissociation $\mathrm{H}_{2} \mathrm{CO} \rightarrow \mathrm{H}_{2}+\mathrm{CO}$. The upper figure is a contour plot of the potential as a function of the reaction coordinate $s$ and the out-of-plane bend coordinate $Q$. The lower figure is the potential along the reaction path, i.e., along the line $Q=$ 0 of the upper figure. The barrier height for this reaction is $\sim 80-90 \mathrm{kcal} / \mathrm{mol}$.

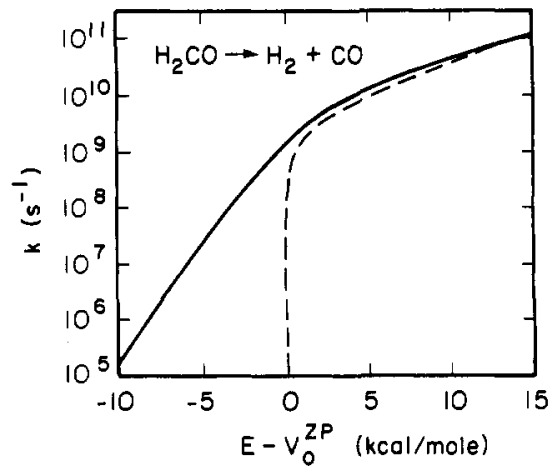

Figure 3. The microcanonical unimolecular rate constant for the formaldehyde dissociation $(J=0)$ as a function of energy relative to the classical threshold. The solid line includes the effects of tunneling, and the broken line is the classical result that neglects tunneling. [If $E$ is the total energy in the molecule $\left(E=E_{\text {ex }}+\right.$ $E_{\mathrm{zp}}$, where $E_{\mathrm{zp}}$ is the zeroth point energy of $\mathrm{H}_{2} \mathrm{CO}$ and $E_{\mathrm{ez}}$ the excitation energy), then $V_{0}^{\mathrm{zp}}=V_{0}+E_{2 \mathrm{p}}^{*}$, where $V_{0}$ is the "bare" barrier height and $E_{\mathrm{zp}}{ }^{*}$ the zero-point energy of the transition state. Thus $E-V_{0}{ }^{\mathrm{zp}}=E_{\mathrm{ex}}-\left(V_{0}+E_{\mathrm{zp}}{ }^{*}-E_{\mathrm{zp}}\right)$.]

constant $k(E, J)$ for $J=0$ as a function of total energy $E$ (relative to the classical threshold).$^{18}$ The dashed line in Figure 3 shows, for comparison, the classical RRKM rate constant. One sees that the rate is somewhat greater than $10^{9} \mathrm{~s}^{-1}$ at the classical threshold and is $\sim 10^{5} \mathrm{~s}^{-1}$ at an energy $\sim 10 \mathrm{kcal} / \mathrm{mol}$ below the classical threshold. Similar calculations have been carried out for $J>0 ;{ }^{18,24}$ the rate in general decreases with increasing $J$, which is understandable since increasing $J$ takes energy out of the reaction coordinate.

The most dramatic aspect of tunneling will thus be manifested if one excites the isolated molecule to an energy below the classical threshold. Without inclusion of tunneling the unimolecular decay rate is zero, while tunneling allows a finite rate, which this example shows can be physically significant for energies as much as 10 $\mathrm{kcal} / \mathrm{mol}$ below the classical threshold.

Experiments on formaldehyde are actually accomplishing this, though the interpretation is far from straightforward. The most relevant experiments excite $\mathrm{H}_{2} \mathrm{CO}$ to individual rotational-vibrational states of $\mathrm{S}_{1}$, the first electronically excited singlet state; this is followed by a radiationless transition $\mathrm{S}_{1} \rightarrow \mathrm{S}_{0} *$ (highly vibrationally excited), and it is $\mathrm{S}_{0} *$ which undergoes 


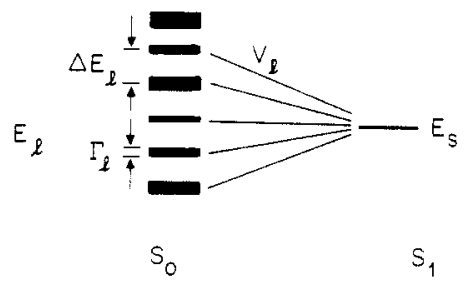

Figure 4. Schematic picture of the photochemical dissociation of formaldehyde; cf. eq 2.13 and 2.14). $E_{\mathrm{s}}$ is the energy of the state $|s\rangle$ of $\mathrm{S}_{1}$ that is excited by the laser, $V_{l}$ are the nonadiabatic couplings between $|s\rangle$ and the various states $|l\rangle$ of $S_{0}$, and $\left\langle\Gamma_{l}\right|$ are the "widths" of these states with respect to unimolecular decomposition. $\Delta E_{l}$ is the spacing of the levels $E_{l}$.

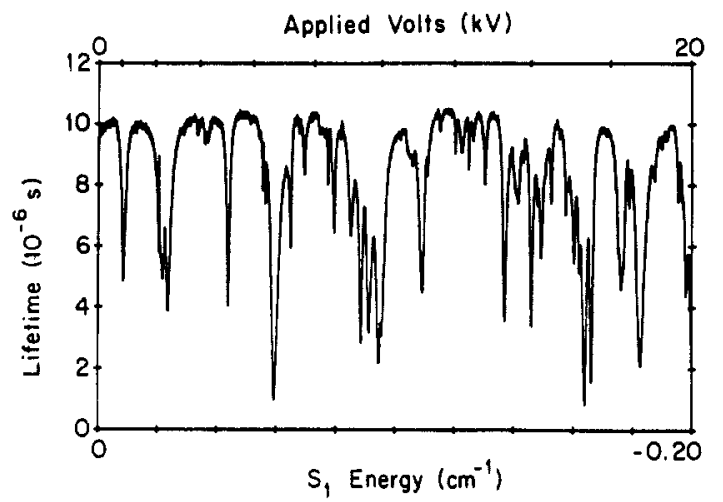

Figure 5. Lifetime of the $1_{11}(M=1)$ rotational state of the $4^{1}$ vibrational state of $\mathrm{S}_{1} \mathrm{D}_{2} \mathrm{CO}$, as a function of the energy of this level as tuned by an applied electric (Stark) field, from ref 30. The zero of energy here is an excitation energy of $28375 \mathrm{~cm}^{-1}=$ $81.1 \mathrm{kcal} / \mathrm{mol}$.

unimolecular decomposition. The reader should see ref 4 for a more complete discussion of these experiments.

Figure 4 shows a schematic picture of this mechanism. The widths $\left\{\Gamma_{l}\right\}$ of the individual highly excited vibrational states of $\mathrm{S}_{0}$ give the state-specific unimolecular decay rates $k_{l}$

$$
k_{l} \equiv \Gamma_{l} / \hbar
$$

and they are the objects of interest with regard to discussing the unimolecular reaction dynamics in the ground electronic state. The quantity directly measured in these experiments, however, is the decay rate (i.e., inverse lifetimes) of the initially excited state $|s\rangle$ of $S_{1}$, and within a perturbative approximation to the $S_{1}-S_{0}$ coupling it is given by ${ }^{25}$

$k_{s}=\hbar^{-1} \sum_{l}\left|V_{s l}\right|^{2} \Gamma_{l} /\left[\left(E_{l}-E_{s}\right)^{2}+\left(\Gamma_{l} / 2\right)^{2}\right]$

It is thus not trivial to obtain the individual widths $\left\{\Gamma_{l}\right\}$ from such measurements. In fact, if many terms contribute to the sum in eq 2.13 , then it is well-approximated by

$$
k_{s}=\frac{2 \pi}{\hbar} \sum_{l}\left|V_{s l}\right|^{2} \delta\left(E_{s}-E_{l}\right)
$$

so that no information about $\left\{\Gamma_{l}\right\}$ is obtainable. Nonperturbative treatments of the decay can lead to nonexponential decay (i.e., multiple exponential decay with quantum beats), and if these features are observed, it is possible to extract not only the energies $\left\{E_{l}\right\}$ and widths $\left\{\Gamma_{l}\right\}$ but also the nonadiabatic coupling matrix elements $V_{s l}{ }^{26}$

A major breakthrough occurred when Weisshaar and Moore ${ }^{27-29}$ first carried out these experiments in a

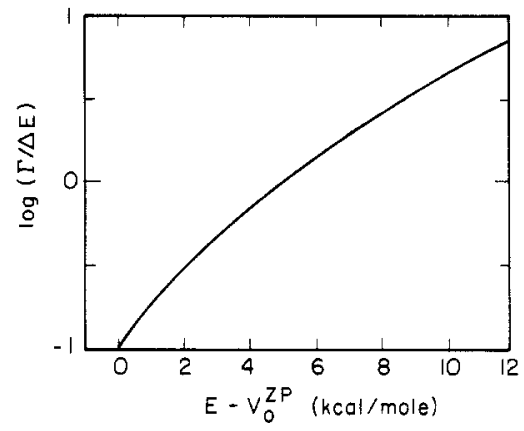

Figure 6. Average width-to-spacing ratio for levels in $\mathrm{S}_{0} \mathrm{H}_{2} \mathrm{CO}$ $(J=0)$, as a function of energy relative to the classical threshold. (See Figure 3 for a discussion of the abscissa.)

TABLE I. Experimentally Determined Energies and Unimolecular Dissociation Rates for Individual Quantum States of $S_{0}$ Formaldehyde

\begin{tabular}{crcc}
\hline ref & $J$ & $\begin{array}{c}E_{\text {ex }}{ }^{a} \\
\mathrm{kcal} / \mathrm{mol}\end{array}$ & $\begin{array}{c}\text { rate } \\
\left(10^{7} \mathrm{~s}^{-1}\right)^{b}\end{array}$ \\
\hline 27 & 5 & 81.40 & 5.6 \\
27 & 10 & 82.70 & 5.0 \\
27 & 11 & 82.79 & 5.0 \\
27 & 4 & 81.37 & 530 \\
29 & 2 & 80.94 & 7.1 \\
29 & 2 & 81.03 & 2.8 \\
31 & 1 & 80.98 & 17 \\
31 & 1 & 80.98 & 5.6 \\
31 & 1 & 80.98 & 1.3
\end{tabular}

${ }^{a}$ This is the excitation energy above the ground state of formaldehyde. ${ }^{b}$ The analysis is still tentative in some cases, so that these values should be considered preliminary.

variable electric (i.e., Stark) field, which modulates the level $E_{s}$ relative to the $\mathrm{S}_{0}$ levels $\left\{E_{l}\right\}$. Figure 5 shows an example ${ }^{30}$ of these experimental results, the decay rate $k_{s}$ as a function of the electric field strength, for a particular ro-vibrational state of $\mathrm{S}_{1}$ of $\mathrm{D}_{2} \mathrm{CO}$. The observed structure is assumed ${ }^{4}$ to be due to the fact that the levels of $S_{0}$ * constitute a "lumpy continuum"; i.e., the broadened levels $\left\{E_{l}\right\}$ are sufficiently sparse (cf. Figure 4) that they do not overlap and form a "smooth continuum" into which the state $|s\rangle$ decays.

From the transition-state theory rate expression one can actually obtain a useful quantitative measure of the "lumpy continuum" character of the $\mathrm{S}_{0}$ * states. The average width $\bar{\Gamma}_{l}$ is given in terms of the average rate $k$ of eq 2.1

$$
\bar{\Gamma}_{l} \equiv \hbar k(E, J)
$$

and the average level spacing $\Delta \bar{E}_{l}$ of $\mathrm{S}_{0}^{*}$ states is the reciprocal of the density of states $\rho$

$$
\Delta \bar{E}_{l} \equiv 1 / \rho(E, J)
$$

Thus the average width-to-spacing ratio is given by

$$
\frac{\bar{\Gamma}_{l}}{\Delta \bar{E}_{l}}=\frac{1}{2 \pi} \sum_{n, K} P\left(E-\epsilon_{n J K}^{*}\right)
$$

This quantity is shown in Figure 6, as a function of energy relative to the classical threshold, for $J=0$. If one calls the continuum "lumpy" if

$$
\frac{\bar{\Gamma}_{l}}{\Delta \bar{E}_{l}} \leq 1 / 2
$$

then one sees from Figure 6 that the $S_{0}$ * continuum is still lumpy (for $J=0$ ) for energies up to $\sim 3 \mathrm{kcal} / \mathrm{mol}$ 


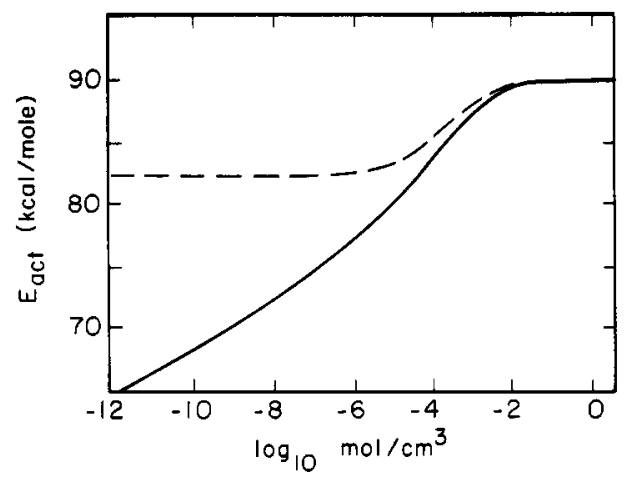

Figure 7. Activation energy for the effective unimolecular rate constant (see eq 2.19 and 2.21 ) as a function of density $(n / V)$ (which is proportional to pressure via $p V=n R T$ ). The solid curve includes the effect of tunneling in $k(E)$, and the dashed curve neglects it.

above the classical threshold for the reaction.

Table I lists some energies $\left\{E_{l}\right\}$ and decay rates $\left\{\Gamma_{l} / \hbar\right\}$ for individual ro-vibrational states of $S_{0}$ states that have been determined ${ }^{27,29,31}$ by using the above Stark shift measurements. The agreement with the values computed via the statistical approximation (Figure 3) is in general quite good, demonstrating that at least the order of magnitude given by the tunneling mechanism is correct. Perhaps even more interesting, however, is that the experiments appear to demonstrate state specificity; i.e., the rate is not a smooth, monotonically increasing function only of the total energy. State specificity will be discussed more fully in section III.

Finally, to conclude this discussion of the formaldehyde dissociation within the statistical approximation, it should be noted that Forst ${ }^{32}$ has considered the effect of tunneling on the traditional thermal decomposition of formaldehyde. Here the effective, pressure-dependent unimolecular rate constant is ${ }^{1,2}$

$k_{\text {uni }}=\frac{1}{Q} \int_{0}^{\infty} \mathrm{d} E \rho(E) e^{-E / k T} \omega k(E) /[\omega+k(E)]$

where $Q$ is the partition function of reactants

$$
Q=\int_{0}^{\infty} \mathrm{d} E \rho(E) e^{-E / k T}
$$

and $\omega$ is the collision frequency (proportional to pressure). $k(E)$ is the microcanonical unimolecular rate constant (eq 2.1), for $J=0$. Forst finds that tunneling causes the effective activation energy

$$
E_{\text {act }} \equiv-\frac{\mathrm{d}}{\mathrm{d}(1 / k T)} \ln k_{\text {uni }}
$$

to fall off "forever" with decreasing pressure; cf. Figure 7. He concludes that the thermal decomposition experimental data are consistent with the photochemical experiments discussed above and in particular with the notion that the decomposition into $\mathrm{H}_{2}+\mathrm{CO}$ proceeds by tunneling.

\section{Effect of Reaction Path Curvature}

The treatment of tunneling described in section IIA ignores any coupling of reaction coordinate motion to other degrees of freedom in the molecule. Though this may often be reasonable - more detailed calculations for the formaldehyde dissociation ${ }^{33}$ discussed in section IIC, for example, suggests that the error introduced there is no more than a factor of 2-there are some cases where the error this causes can be an order of magnitude or greater. For a standard bimolecular test reaction

$$
\mathrm{H}+\mathrm{H}_{2} \rightarrow \mathrm{H}_{2}+\mathrm{H}
$$

the error is a factor of $\sim 50-100$ in the important threshold region. ${ }^{34}$

There are a variety of still relatively simple models for including, at least approximately, the effect of coupling of the reaction coordinate to other degrees of freedom of the molecular system, i.e., the effect of nonseparability of the reaction coordinate. Most of these are derived from a "reaction path" description of the process, ${ }^{35-37}$ whereby the reaction coordinate $s$ is defined as the distance along the steepest descent path in mass-weighted Cartesian coordinates - the reaction path-that passes through the transition state from reactants to products. The other internal (i.e., vibrational) coordinates of the system are chosen as local harmonic normal modes that are orthogonal to the reaction path. The Hamiltonian of the system in these coordinates is of the form, for $J=0^{36}$

$$
\begin{aligned}
H\left(p_{s}, s, P, Q\right)= & \frac{1 / 2\left[p_{s}-\sum_{k, k^{\prime}=1}^{F-1} Q_{k} P_{k^{\prime}} B_{k, k^{\prime}}(s)\right]^{2}}{\left[1+\sum_{k=1}^{F-1} Q_{k} B_{k, F}(s)\right]^{2}} \\
& +V_{0}(s)+\sum_{k=1}^{F-1}\left(1 / 2 P_{k}^{2}+1 / 2 \omega_{k}(s)^{2} Q_{k}^{2}\right)
\end{aligned}
$$

where $\left(Q_{k}, P_{k}\right)(k=1, \ldots, F-1)$ are the coordinates and momenta for the local harmonic modes perpendicular to the reaction path, $V_{0}(s)$ is the potential energy along the reaction path, and the functions $\left\{B_{k, F}(s)\right\}(k=1, \ldots$, $F-1)$ describe coupling of the vibrational modes to the reaction coordinate (labeled as mode $F$ ); the functions $\left\{B_{k, k^{\prime}}(s)\right\}\left(k, k^{\prime}=1, \ldots, F-1\right)$ describe coupling of the modes among themselves. This reaction path Hamiltonian $^{36}$ and its applications have been reviewed several times ${ }^{34 b, 38,39}$ recently, and the reader should see these for more information.

The primary effect which couples the reaction coordinate $s$ to the other degrees of freedom is the curvature of the reaction path, which is characterized by the curvature coupling functions $\left\{B_{k, F}(s)\right\}$. One can show that the total curvature $\kappa(s)$ of the reaction path in the $F$-dimensional space at distance $s$ along it is

$$
\kappa(s)=\left[\sum_{k=1}^{F-1} B_{k, F}(s)^{2}\right]^{1 / 2}
$$

The individual coupling functions $B_{k F}(s)$ are a measure of how the total curvature of the reaction path projects locally onto the various modes $k$ orthogonal to it. It is this curvature of the reaction path which causes the coupling. Synonymous with the effect of nonseparability of the reaction coordinate on tunneling, therefore, is the effect of reaction path curvature on tunneling.

The simplest approximate expression which takes account of reaction path curvature on the tunneling probability was first suggested by Marcus and Coltrin ${ }^{40}$

$$
P_{n}(E)=e^{-2 \theta_{0}(E)} \prod_{k=1}^{F-1} e^{2 \theta_{k}(E)}
$$

and also results from other theoretical approaches. ${ }^{34 b, 41}$ 
Here $\theta_{0}$ is the vibrationally adiabatic action integral

$$
\theta_{0}=\int_{s_{\varsigma}}^{s_{\gamma}} \sqrt{2\left[V_{\mathbf{n}}(s)-E\right]}
$$

where

$$
V_{\mathbf{n}}(s)=V_{0}(\mathrm{~s})+\sum_{k=1}^{F-1} \hbar \omega_{k}(s)\left(n_{k}+1 / 2\right)
$$

i.e., the factor $e^{-2 \theta_{0}}$ in eq 2.24 is the zeroth-order tunneling probability. The action integrals $\left\{\theta_{\mathrm{k}}\right\}$ contain the influence of reaction path curvature

$$
\theta_{k}=\left|\int_{s_{\zeta}}^{s_{1}} \mathrm{~d} s \sqrt{2\left[V_{\mathbf{n}}(s)-E\right)}\left[\frac{2 n_{k}+1}{\omega_{k}(s)}\right]^{1 / 2} B_{k, F}(s)\right|
$$

and one sees that the factors $\left\{e^{2 \theta_{k}}\right\}$ in eq 2.24 in general increase the tunneling probability beyond what it would be if reaction path curvature were ignored.

For the well-studied test case $\mathrm{H}+\mathrm{H}_{2} \rightarrow \mathrm{H}_{2}+\mathrm{H}$, this simple model (eq 2.24-2.26) corrects the tunneling probability from being a factor of 50-100 too small if reaction path curvature is ignored to within a factor of 2 of the correct value. ${ }^{34 b, 40}$ If the curvature correction of the tunneling probability is smaller than this, then one has some confidence that this model will be able to described it at least semiquantitatively. As noted above, the curvature correction to the tunneling probability given by eq $2.24-2.26$ for the formaldehyde dissociation is less than a factor of 2 .

Other, more sophisticated and accurate models ${ }^{42,43}$ for describing the effects of reaction path curvature are described in Schatz' paper ${ }^{12}$ in this volume on tunneling in bimolecular reactions. They can also be applied to the present unimolecular case.

\section{State-Speciflc Unimolecular Reactions}

As discussed in section IIB, the "ultimate" description of a unimolecular reaction, theoretically and experimentally, is the determination of the unimolecular rate constants for individual quantum states of the reactant molecule, i.e., the determination of the energies and lifetimes of the "metastable eigenstates". (These are solutions of the Schrödinger equation with outgoing wave boundary conditions and thus have complex eigenvalues $E_{l}-i \Gamma_{l} / 2$.) Only then can one answer the question of whether a particular unimolecular reaction is intrinsically statistical, case a of section IIB, or state-specific, case b of section IIB.

[It is interesting to point out that it is in the tunneling region where it should be easiest to determine statespecific unimolecular rates. This follows from the discussion related to eq $2.15-2.18$ of section IIC, which showed that it is the tunneling region where the average width $\bar{\Gamma}_{l}$ of the eigenmetastable states is less than their average spacing $\Delta \bar{E}_{l}$. With a sufficiently narrow laser, therefore, it is straightforward (in principle) to excite individual eigenmetastable states since they do not overlap.]

To illustrate state specificity, particularly for a tunneling system, it is useful to consider the two-oscillator model system discussed in section IIB. Figure 8 shows a plot of the calculated unimolecular decay rates of the eigenmetastable states vs. their energies for the case

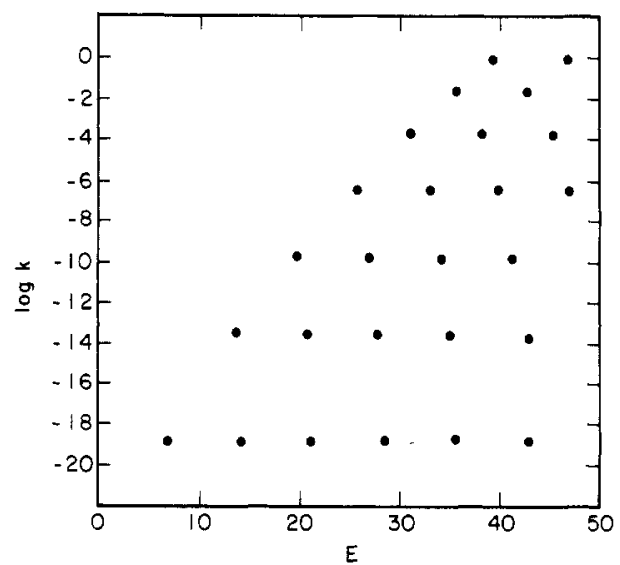

Figure 8. State-specific rate constants $k \equiv \Gamma / \hbar$ for unimolecular decay vs. total energy $E$, for the two-oscillator model with the Hamiltonian of eq 2.6. This case is for no coupling between the two modes.

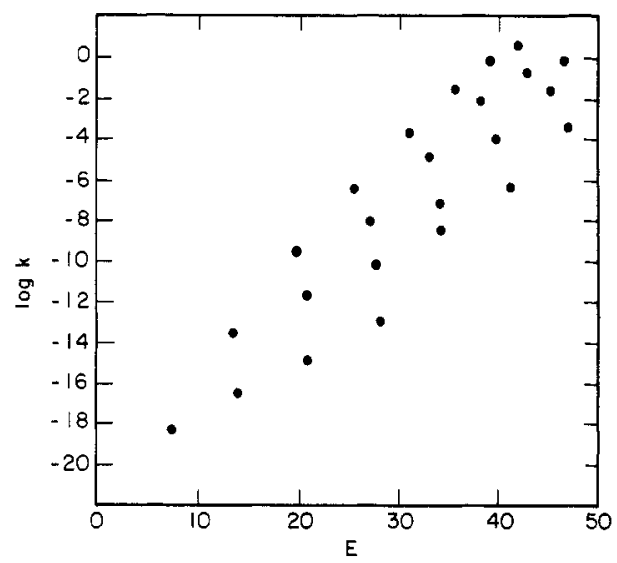

Figure 9. Same as Figure 8, except for the addition of a coupling term to the Hamiltonian of eq 2.6. See ref 23 for the specific form of the coupling.

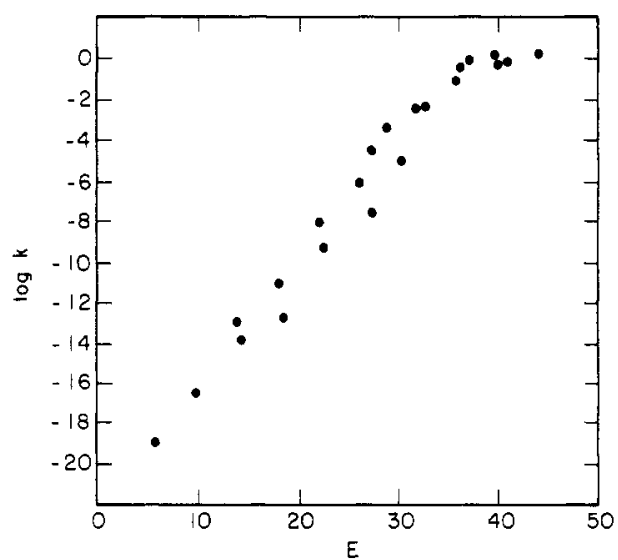

Figure 10. Same as Figure 9, with a stronger coupling.

that the two oscillators are uncoupled, i.e., for the Hamiltonian of eq 2.6. This shows the extreme limit of state-specificity; i.e., states with essentially the same energy have vastly different decay rates.

[The reader is referred to the original paper ${ }^{23}$ for the details of these calculations. They are rather straightforward quantum mechanical basis set calculations, and they use the complex scaling method ${ }^{44}$ to determine the energies and decay rates. Such "brute force" quantum mechanical calculations are only feasible for two or perhaps three vibrational degrees of freedom.] 


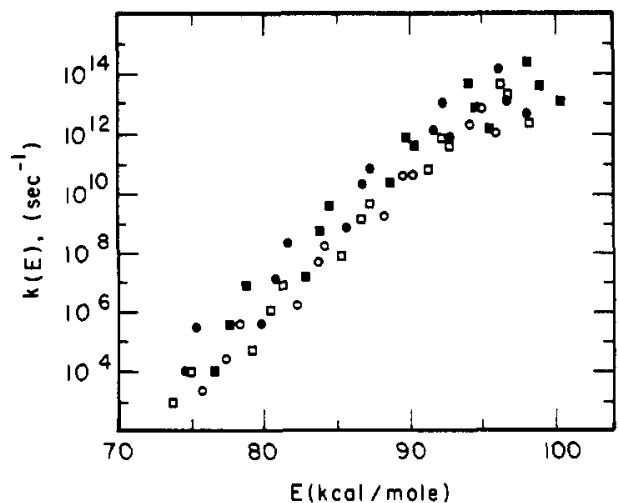

Figure 11. State-specific unimolecular rate constants for a two-mode model of the formaldehyde dissociate $\mathrm{H}_{2}+\mathrm{CO}$. See ref 26. The solid squares and circles are states of $A_{1}$ and $B_{1}$ symmetry, respectively, and open squares and circles are $A_{2}$ and $\mathrm{B}_{2}$ states.

Figure 9 shows how the rate vs. energy plot changes if a nonseparable term is added to the Hamiltonian which couples the $x$ and $y$ degrees of freedom, and Figure 10 shows the most statistical-like result that was obtained when the coupling term and relative $x$ and $y$ frequencies were chosen to produce the most effective mixing. This latter case is beginning to approach the ideal limit of intrinsic statistically behavior, when the individual rates $\left\{k_{l}\right\}$ are a smooth function only of their energy $\left\{E_{l}\right\}$

$$
k_{l}=k\left(E_{l}\right)
$$

Here $k(E)$ is (by definition) the statistical rate, the average rate at energy $E$. Even in Figure 10, though, one sees that there remains a significant degree of state specificity; rates for states of essentially the same energy vary approximately 1 order of magnitude.

Similar calculations have been carried out for a two-mode model of the formaldehyde decomposition, ${ }^{26}$ the two modes being the reaction coordinate and the out-of-plane bend; cf. the potential surface in the top part of Figure 2. The out-of-plane bend was chosen because it is the mode least strongly coupled to the reaction coordinate (via the reaction path Hamiltonian as described in section IID) and the one thus most likely to show significant state specificity. Figure 11 shows the results of this calculation. The overall trend follows the statistical rate very well, but one sees that there is a significant degree of state specificity: rates vary by approximately 2 orders of magnitude for various states with essentially the same total energy. Interestingly, this is roughly the same degree of variation seen in the state-specific unimolecular decay rates that have been determined experimentally; cf. Table I.

How should one interpret the various degrees of state specificity illustrated by Figures 8-11? One notion that occurred to us ${ }^{23}$ early-which actually turns out not to be very useful in this case-was to see how state specificity in the eigenmetastable states correlates with chaotic dynamics ${ }^{10 b, c}$ in the (classical) intramolecular dynamics. The idea was that chaotic dynamics should correlate with statistical behavior of the rates, while quasiperiodic dynamics should correlate with state specificity. If this were true, then one should see state specificity at low energies and then a more-or-less abrupt transition energy above which the rates would be more statistical-like. This behavior was never observed,

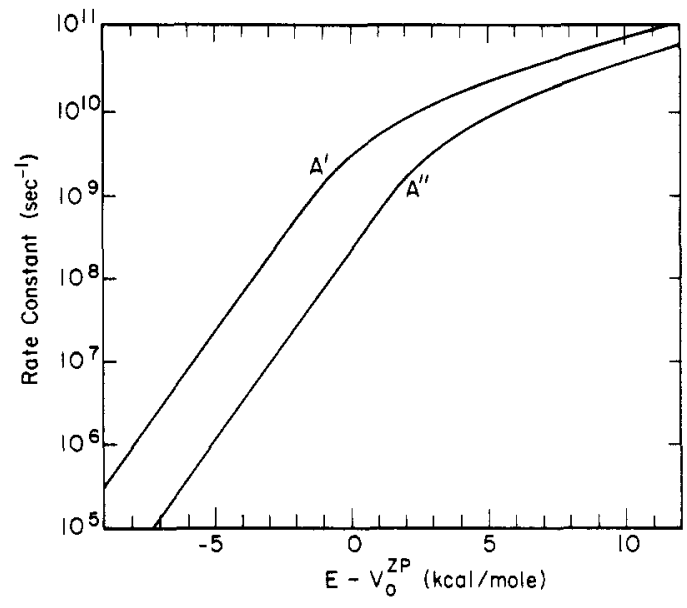

Figure 12. Microcanonical rate constant for the reaction $\mathrm{H}_{2} \mathrm{CO}$ $\rightarrow \mathrm{H}_{2}+\mathrm{CO}(J=0)$ for the symmetry $\mathrm{A}^{\prime}$ and $\mathrm{A}^{\prime \prime}$ as a function of total energy relative to the zero-point energy adjusted barrier height.

even though the classical dynamics of some of these systems do show transitions from quasiperiodic dynamics to chaotic dynamics. (It should be noted, though, that in other kinds of studies ${ }^{45}$ there have been strong correlations seen between predissciation rates and the underlying quasiperiodic-chaotic character of the classical mechanics.)

Another way to think about state specificity in the unimolecular rate constants is to suppose that there are some approximately conserved quantum numbers which are operative. Total angular momentum, for example, is a rigorously conserved quantum number (in free space, i.e., no electronic or magnetic fields), and as was done in section II one applies the statistical approximation separately for each value of $J$ when calculating the average rate constant at energy $E$. There is thus a family of $k$ vs. $E$ curves, a separate one for each value of $J$. If there are other good (or approximately good) quantum numbers $\lambda$ by which the energy levels of the reactant molecule and the transition state can be labeled, then here too one should apply the statistical approximation separately for each value of $\lambda$, i.e., eq 2.1 should be modified to read

$$
\begin{array}{r}
k_{\lambda}(E, J)=\left[2 \pi \hbar \rho_{\lambda}(E, J)^{-1} \sum_{(\mathbf{n}, K)_{\lambda}} P\left(E-\epsilon_{\mathbf{n} J K}{ }^{*}\right)\right] \\
\rho_{\lambda}(E, J)=\sum_{(\mathbf{n}, K)_{\lambda}} \delta\left(E-\epsilon_{\mathbf{n} J K}\right)
\end{array}
$$

where the notation $(\mathbf{n}, K)_{\lambda}$ means that only vibrational-rotational states of type $\lambda$ are included in the sum.

The calculated results of the two-dimensional model problems in Figures 8-11 do suggest this behavior. Certainly the separable limit, Figure 8 , shows it since here the quantum numbers $n_{x}$ and $n_{y}$ are separately conserved. But even in the nonseparable cases these rates seem to fall on several separate $k$ vs. $E$ curves which could be labeled by a discrete index $\lambda$.

Another example of conserved quantum numbers is seen in the formaldehyde dissociation for $J=0$. Because the reaction path ${ }^{33}$ is planar, the $A^{\prime} / A^{\prime \prime}$ symmetry (even/odd on reflection in the plane) is conserved during the dissociation dynamics, so the statistical approximation should be applied separately for each symmetry. ${ }^{46}$ Figure 12 shows these two rates, and one sees that in the tunneling region this symmetry-induced state specificity accounts for about 1 order of magnitude 


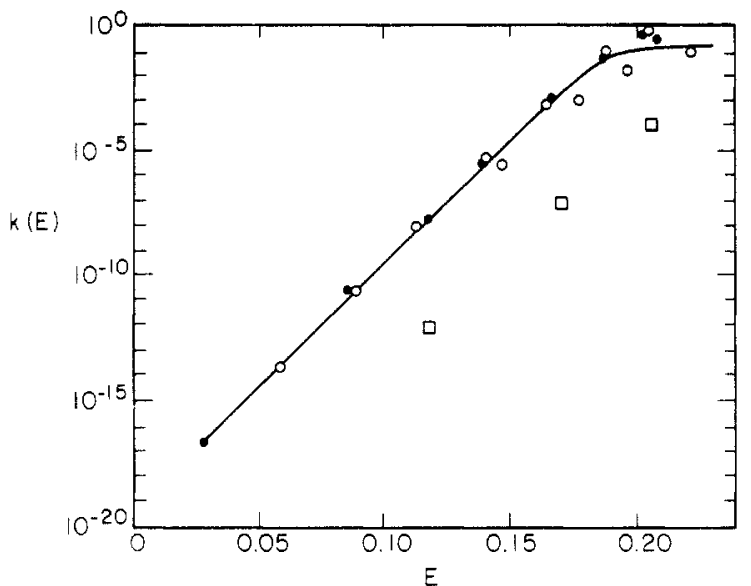

Figure 13. State-specific rate constants for unimolecular decay in the Henon-Heiles potential $V(x, y)=1 /{ }_{2} x^{2}-1 /{ }_{3} x^{3}+1 / 2 y^{2}+$ $x y^{2}$, as a function of energy; see ref 47 for complete details. Solid points, squares, and circles denote states of symmetry $A_{1}, A_{2}$, and $\mathrm{E}$, respectively. The solid curve is the result of the microcanonical transition state theory (eq 2.1).

variation in the rate constants for states with the same energy.

A final example of state specificity is seen in calculations carried out by Waite et al..$^{47}$ and Taylor et al. ${ }^{48}$ on another two-dimensional model potential, the well-studied Henon-Heiles potential. Figure 13 shows an example of these results; this potential has $C_{3 v}$ symmetry, and the $A_{1}, A_{2}$, and $E$ states are indicated separately. One sees a large symmetry-induced effect; i.e., the $A_{2}$ rates are much less than the $A_{1}$ and $E$ rates (for the same energy). Beyond this, one also sees about an order of magnitude of state specificity among the $E$ states themselves (the open circles) and a hint at some state specificity among the highest energy $\mathrm{A}_{1}$ states. Taylor et al. are able to explain some of this state specificity, at least qualitatively, by using ideas of Hose and Taylor ${ }^{49}$ to classify various states. The basic idea is that many of the states correspond to quasiperiodic motion, i.e., have approximately conserved quantum numbers and thus do not explore all the energetically accessible space. Some thus correspond to motion that avoids the transition-state region, while others correspond to motion that is localized in its vicinity, and these states decay slower and faster, respectively, than the average.

To summarize, state specificity in unimolecular reaction rates has been seen in a variety of rigorous quantum mechanical calculations for model systems with two degrees of freedom. In fact, for none of these model problems has one seen any less than approximately 1 order of magnitude in variation of the unimolecular rates about their average rate at a given energy. The formaldehyde dissociation is the best studied system experimentally, and though the analysis is not completely settled, it appears that states of essentially the same energy (and symmetry) have rates that vary by 1-2 orders of magnitude. It may turn out-and this is in fact my belief - that essentially all unimolecular reactions will show a significant degree of state specificity at this most microscopic level of individual quantum states.

To model state-specific unimolecular rate constants requires theoretical treatments that go beyond the statistical approximations described in section II, and this is very challenging. Such calculations have so far been carried out only for two-dimensional $\mathrm{n}$ del problems. The reason that statistical approximations have been and continue to be so useful, of course, is that most experiments do not determine the rates for individual quantum states but rather an average over many of them, and for these average rates statistical approximations are quite adequate. In any event they are adequate for determining the average rate even if one has available state-specific rates.

\section{Concluding Remarks}

Statistical approximations do a very good job of describing the average unimolecular reaction rate for a molecule with energy $E$ (and angular momentum J), even in cases where the reaction proceeds by tunneling. Sometimes it is necessary to take account of nonseparability of the reaction coordina' : from the other degrees of freedom of the molecule (1.e., reaction path curvature), but there are a variety of relatively simple models that describe this reasonably well.

In the tunneling region, where the widths $\left\{\Gamma_{l}\right\}$ of individual eigenmetastable states are less than their average spacing $\Delta \bar{E}_{l}$, it is meaningful to consider the unimolecular reaction rates $k_{l} \equiv \Gamma_{l} / \hbar$ of these individual quantum states. At this most fine-grained level, theoretical calculations on various model problems show a significant degree of state specificity; i.e., rates fluctuate approximately 1 order of magnitude or more about the average rate at given energy. (The average rate is well-approximated by statistical theory.) Experimental results on the unimolecular decomposition of formaldehyde appear to show state specificity; i.e., rates for individual quantum states of essentially the same energy (and angular momentum) vary 1-2 orders of magnitude.

As high resolution laser studies of molecular spectroscopy and dynamics continue to develop, one expects to see more experimental examples of state specificity in various aspects of reaction dynamics. This will provide a rigorous challenge for theoretical models and methods.

Acknowledgments. This work has been supported by the Director, Office of Energy Research, Office of Basic Energy Sciences, Chemical Sciences Division of the U.S. Department of Energy, under Contract DE-AC0376 SF00098 and also in part by the National Science Foundation Grant CHE84-16345. I wish to thank Drs. H. L. Dai, C. B. Moore, W. Polik, and R. W. Field for helpful discussions regarding their experimental results on photodissociation of formaldehyde. Special thanks are due H. L. Dai for making available some of the values in Table I (ref 31) and W. Polik for providing Figure 5 (ref 30 ), both prior to publication.

\section{Reterences}

(1) P. J. Robinson and K. A. Holbrook, "Unimolecular Reactions", Wiley, New York, 1972.

(2) W. Forst, "Theory of Unimolecular Reactions", Academic Press, New York, 1973.

(3) F. F. Crim, Annu. Rev. Phys. Chem., 35, 657 (1984).

(4) C. B. Moore and J. C. Weisshaar, Annu. Rev. Phys. Chem., 34, 525 (1983).

(5) Note, for example the International CECAM Workshop on Intramolecular Vibrational Redistribution and Quantum Chaos, University of Rochester, October 3-5, 1985.

(6) C. E. Dykstra, Annu. Rev. Phys. Chem., 32, 25 (1981). 
(7) See, for example, the papers from the Fifth American Conference on Theoretical Chemistry, June 15-20, 1984, in $J$. Phys. Chem., 89, 2121-2234 (1985).

(8) S. L. Baughcum, Z. Smith, E. B. Wilson, Jr., and R. W. Duerst, J. Am. Chem. Soc., 106, 2265 (1984).

(9) T. Carrington, Jr., and W. H. Miller, J. Chem. Phys., 84, 4364 (1986).

(10) See, for example, (a) A. H. Zewail, Ed., "Advances in Laser Chemistry", Springer, New York, 1978. (b) D. W. Noid, M. L. Koszykowski, and R. A. Marcus, Annu. Rev. Phys. Chem., 32 267 (1981); (c) E. B. Stechel and E. J. Heller, Annu. Rev. Phys. Chem., 35, 563 (1984); (d) V. E. Bondybey, Annu. Rev. Phys. Chem. 35, 591 (1984).

(11) K. G. Kay, J. Chem. Phys., 64, 2112 (1976); 65, 3813 (1976); 68, 434 (1978); R. W. Numrich and K. G. Kay, ibid., 70, 4343 (1979); 71, 5352 (1979).

(12) G. C. Schatz, this volume.

(13) D. G. Truhlar and B. C. Garrett, Annu. Rev. Phys. Chem., 35, 159 (1984).

(14) W. Siebrand, T. A. Wildman, and M. Z. Zgierski, J. Am. Chem. Soc., 106, 4083 (1984).

5) R. A. Marcus and O. K. Rice, J. Phys. Colloid Chem., 55, 894 (1951); R. A. Marcus, J. Chem. Phys., 20, 359 (1952); 43, 2658 (1965).

(16) (a) W. H. Miller, Acc. Chem. Res., 9, 306 (1976); (b) P. Pechukas, Annu. Rev. Phys. Chem., 32, 159 (1981); (c) D. G. Truhlar, W. L. Hase, and J. T. Hynes, J. Phys. Chem., 87, 2664 (1983).

(17) R. A. Marcus, J. Chem. Phys., 45, 2138 (1966).

(18) W. H. Miller, J. Am. Chem. Soc., 101, 6810 (1979).

(19) E. Wigner, Trans. Faraday Soc., 34, 29 (1938).

(20) J. C. Keck, Adv. Chem. Phys., 13, 85 (1967); Adv. At. Mol. Phys., 8, 39 (1972).

(21) W. H. Wang and R. A. Marcus, J. Chem. Phys., 55, 5625 (1971).

(22) M. Quack and J. Troe, Ber. Bunsenges. Phys. Chem., 78, 240 (1974).

(23) B. A. Waite and W. H. Miller, J. Chem. Phys., 73, 3713 (1980).

(24) J. Troe, J. Phys. Chem., 88, 4375 (1984).

(25) See, for example, W. M. Gelbart, K. C. Freed, and S. A. Rice, J. Chem. Phys., 52, 2460 (1970); P. Avouris, W. M. Gelbart, and M. A. El-Sayed, Chem. Rev. 77, 793 (1977)

(26) B. A. Waite, S. K. Gray, and W. H. Miller, J. Chem. Phys., 78, 259 (1983).

(27) J. C. Weisshaar and C. B. Moore, J. Chem. Phys., 72, 2875, $5415(1980)$

(28) W. E. Henke, H. L. Selzle, T. R. Hays, E. W. Schlag, and S. H. Lin, J. Chem. Phys., 76, 1335 (1982).

(29) H. L. Dai, R. W. Field, and J. L. Kinsey, J. Chem. Phys., 82, 1606 (1985)

(30) D. R. Guyer, W. F. Polik, and C. B. Moore, J. Chem. Phys. 84, 6519 (1986).
(31) A. Ritter, W. Merry, D. Frye, and H. L. Dai, private communication.

(32) W. Forst, J. Phys. Chem., 87, 4489 (1983).

(33) S. K. Gray, W. H. Miller, Y. Yamaguchi, and H. F. Schaefer, J. Am. Chem. Soc., 103, 1900 (1981).

(34) (a) R. T. Skodje, D. G. Truhlar, and B. C. Garrett, J. Chem. Phys., 77, 5955 (1982); (b) C. J. Cerjan, S.-h. Shi, and W. H. Miller, J. Phys. Chem., 86, 2244 (1982).

(35) R. A. Marcus, J. Chem. Phys., 49, 2610 (1968).

(36) W. H. Miller, N. C. Handy, and J. E. Adams, J. Chem. Phys., 72, $99(1980)$.

(37) For other papers on reaction path approaches, see (a) G. L. Hofacker, Z. Naturforsch., A: Astrophys., Phys. Phys. Chem. 18A, 607 (1963); (b) S. F. Fischer, G. L. Hofacker, and R. Seiler, J. Chem. Phys., 51, 3941 (1969); (c) S. F. Fischer and M. A. Ratner, J. Chem. Phys., 57, 2769 (1972); (d) P. Russegger and J. Brickman, ibid., 62, 1086 (1976); 60, 1 (1977); (e) M.V. Basilevsky, Chem. Phys., 24, 81 (1977); 67, 337 (1982); M. V. Basilevsky and A. G. Shamov, ibid, 60,347 (1981); (f) K. Fukui, S. Kato, and H. Fujimoto, J. Am. Chem. Soc., 97, I (1975) K. Yamashita, T. Yamabe, and K. Fukui, Chem. Phys. Lett 84, 123 (1981); K. Fukui, Acc. Chem. Res., 14, 363 (1981); (g) K. Ishida, K. Morokuma, and A. Komornicki, J. Chem. Phys. 66, 2153 (1977); (h) A. Nauts and X. Chapuisat, Chem. Phys Lett., 85, 212 (1982); X. Chapuisat, A. Nauts, and G. Durand Chem. Phys., 56, 91 (1981); (i) J. Pancir, Collect. Czech. Chem. Commun., 40, 1112 (1975); 42, 16 (1977); (j) G. A. Natanson, Mol. Phys., 46, 481 (1982).

(38) W. H. Miller in Potential Energy Surfaces and Dynamical Calculations, D. G. Truhlar, Ed.; Plenum Press, New York, $1981, \mathrm{p} 265$.

(39) W. H. Miller, J. Phys. Chem., 87, 3811 (1983).

(40) R. A. Marcus and M. E. Coltrin, J. Chem. Phys., 67, 2609 (1977).

(41) R. T. Skodje and D. G. Truhlar, J. Chem. Phys., 79, 4882 (1983).

(42) B. C. Garrett and D. G. Truhlar, J. Chem. Phys., 79, 4931 (1983).

(43) J. M. Bowman, G.-Z. Ju, and K. T. Lee, J. Chem. Phys., 75 , 5199 (1981); Chem. Phys. Lett., 94, 363 (1983).

(44) See, for example, Int. J. Quantum Chem., 14, No. 4 (1978), the entire volume of which is devoted to the method of complex scaling.

(45) R. L. Sundberg and E. J. Heller, J. Chem. Phys., 80, 3680 (1984).

(46) W. H. Miller, J. Am. Chem. Soc., 105, 216 (1983); J. Chem. Phys., 78, 6640 (1983).

(47) B. A. Waite and W. H. Miller, J. Chem. Phys., 74, 3910 (1981).

(48) Y. Y. Bai, G. Hose, C. W. McCurdy, and H. S. Taylor, Chem Phys. Lett., 99, 342 (1983).

(49) G. Hose and H. S. Taylor, J. Chem. Phys., 76, 5356 (1982). 